\title{
Water Permeability of Pervious Concrete Is Dependent on the Applied Pressure and Testing Methods
}

\author{
Yinghong Qin, ${ }^{1,2}$ Haifeng Yang, ${ }^{1,2}$ Zhiheng Deng, ${ }^{1,2}$ and Jiang $\mathrm{He}^{1,2}$ \\ ${ }^{1}$ College of Civil Engineering and Architecture, Guangxi University, 100 University Road, Nanning, Guangxi 530004, China \\ ${ }^{2}$ Key Laboratory of Disaster Prevention and Engineering Safety of Guangxi, Nanning 530004, China \\ Correspondence should be addressed to Haifeng Yang; yhfgxu@gmail.com
}

Received 18 July 2014; Revised 23 December 2014; Accepted 23 December 2014

Academic Editor: Peter Majewski

Copyright (c) 2015 Yinghong Qin et al. This is an open access article distributed under the Creative Commons Attribution License, which permits unrestricted use, distribution, and reproduction in any medium, provided the original work is properly cited.

\begin{abstract}
Falling head method (FHM) and constant head method (CHM) are, respectively, used to test the water permeability of permeable concrete, using different water heads on the testing samples. The results indicate the apparent permeability of pervious concrete decreasing with the applied water head. The results also demonstrate the permeability measured from the FHM is lower than that from the CHM. The fundamental difference between the CHM and FHM is examined from the theory of fluid flowing through porous media. The testing results suggest that the water permeability of permeable concrete should be reported with the applied pressure and the associated testing method.
\end{abstract}

\section{Introduction}

Pervious concrete is made by eliminating most or all fine aggregates from the concrete mix. Its internal interconnected void space allows storm water to percolate and thus to reduce the amount of run-off. The permeability or the saturated hydraulic conductivity of the pervious concrete signifies its capacity to drain the ponding water from the concrete surface. It quantifies the resistance of the medium to flow and depends only on the characteristics of the porous medium. It is not measured directly but is calculated by measuring its internal states like flux and pressure.

There are several established methods to measure the saturated hydraulic conductivity of porous materials on the basis of Darcy's law. During the measurement, a pervious concrete sample is subjected to a water pressure that is lower sufficiently to support a laminar flow [1-5]. The associated flowing rate is measured to estimate the permeability. The applied pressure during the measurement can be maintained to be constant, referred to as CHM, or be allowed decaying, called FHM [6]. The CHM measures the permeability of pervious concrete by applying a constant water head on the surface of the sample and by weighting the water volume flowing through the sample at a designed time interval $[7,8]$. The FHM allows the water head above the sample surface dropping from a starting level to a designed level and then records the time interval during this dropping process $[9,10]$. As the permeability is not measured directly but is calculated by inverting the applied pressure and its associated water flux, the permeability measured from different scales and distinctive experimental setups is likely to render inconsistent values. It thus merits examining whether the pervious concrete's permeabilities measured from the CHM and the FHM agreed with each other and whether the permeability varies with the applied pressure.

This paper experimentally measures the permeability of pervious concrete under the CHM and the FHM, respectively. Two Portland concrete mixtures are prepared and cored for the water permeability test. The permeability is measured under different water heads to see if the permeability is a function of the applied pressure. The permeability measured from the two methods is compared and it is found that the permeability measured from the FHM tends to be higher than that of the CHM. The fundamental theoretical difference between the two methods is reassessed.

\section{Experiments}

2.1. Sample Preparations. Two concrete mixes consisted of Portland cement, water, and coarse aggregates. Ordinary 


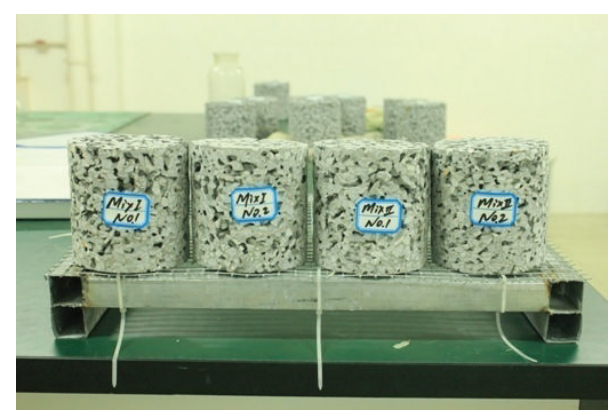

FIGURE 1: Pervious concrete cores for water permeability test.

Type I Portland cement is selected for the experiments. The aggregates used are crushed limestone with a single gradation from $0.5 \mathrm{~cm}$ to $1.0 \mathrm{~cm}$. It has an absorptivity of $0.55 \%$, an observed density of $2725.7 \mathrm{~kg} / \mathrm{m}^{3}$, and a bulk density of $1348.3 \mathrm{~kg} / \mathrm{m}^{3}$. By weight, a cement/aggregate ratio of $1: 4$ is used. One mix has a water-to-cement ratio 0.23 and the other 0.25 . Due to its low water content, $2 \%$ of water-reducing agent with respect to the cement's weight is added to aid the workability of the mixture.

Selected mixtures are mixed by the use of a $0.05 \mathrm{~m}^{3}$ rotating-drum mixer. The aggregate is firstly cast in the mixer, and then the estimated absorbed water is spilled on the aggregate for $1 \mathrm{~min}$ mixture to ensure that the aggregate surface is wet. The cement is afterwards cast to the mixer for another $1 \mathrm{~min}$ mixture with the wet aggregate. Finally, the amount of the water needed for the desired water-to-cement ratio is added to the mixer for another 2 min mixture.

The concrete mixtures are cast in slender wood moulds with a dimension of $150 \times 150 \times 860 \mathrm{~mm}^{3}$. All mixtures are placed by lightly rodding 10 times in the three layers to ensure a uniform compaction in each lift. Mixtures in the mould are leveled by spatulas without any vibration compaction. They are moved to an air-conditioned, foggy room with $20^{\circ} \mathrm{C}$ and with more than $95 \%$ relative humidity for 3 -day curing. After that, they are demolded and the pervious concrete blocks are restored in the air-conditioned room for another 25day curing. Each cured block is drilled for six cores with a diameter of $\Phi 100 \times 100 \mathrm{~mm}$ (Figure 1).

The drilled cores are vacuum-washed to eliminate the drilling-left debris and then are submitted to the basic property's tests such as density and porosity. The porosity of pervious concrete $\varphi$ is determined by

$$
\varphi=1-\frac{\left(w_{2}-w_{1}\right)}{\rho V},
$$

where the $w_{1}(\mathrm{~kg})$ is the sample weight under water; $w_{2}(\mathrm{~kg})$ is the oven-dry sample weight; $\rho\left(\mathrm{kg} / \mathrm{m}^{3}\right)$ is the density of water at room temperature; and $V\left(\mathrm{~m}^{3}\right)$ is the volume of the sample. The sample is oven-dried at $105^{\circ} \mathrm{C}$ for 1.5 hours and subsequently left to cool at room temperature for measuring the oven-dry weight $w_{2}$. The sample's weight $w_{1}$ is measured by submerging and stirring the sample under water for 24 hours to ensure the embedded air bubbles being completely evacuated from the sample. To obtain the volume of the cored sample, a Vernier caliper is used to measure the length and diameter of each sample three times. The average is taken as the dimension of the sample.

\subsection{Water Permeability Measurements}

2.2.1. Falling Head Method (FHM). Permeability of pervious concrete can be tested by a falling head apparatus. The apparatus is schematized as in Figure 2(a). Different apparatus outlines may be used but the mechanism of this pressuredecay method is the same. A faucet supplies sufficient water in the inlet and allows water flow through the sample and finally drains from the outlet. The water head in the inlet is allowed to lift to any desired level and then maintained a steady flow through the pipes. When the test commences, the water-supplied faucet is closed to free the water head in the inlet dropping progressively to level the water head at the outlet. The time interval $\Delta t$ (s) during which the water head falls from $h_{0}(\mathrm{~m})$ to $h_{1}(\mathrm{~m})$ is recorded and the apparent permeability $K_{\text {fal }}\left(\mathrm{m}^{2}\right)$ of the sample can be computed by [11]

$$
K_{\mathrm{fal}}=\frac{\mu L A}{\rho g a \Delta t} \ln \frac{h_{0}}{h_{1}}
$$

where $\mu(\mathrm{Pa} \cdot \mathrm{s})$ is the viscosity of water; $L(\mathrm{~m})$ is the length of the sample; $A\left(\mathrm{~m}^{2}\right)$ is the cross-sectional area of the sample; $a\left(\mathrm{~m}^{2}\right)$ is the cross-sectional area of the water in the falling head pipe.

In this study, the permeability of the sample is determined by the FHM's apparatus like Figure 2(b). The samples are submerged at a stirring water container for 24 hours to ensure that the inner pores are wet sufficiently. The sample is then assembled in the apparatus (Figure 2(b)). Catalyst silicone rubber is used around the perimeter of the sample to prevent water seeping through the side wall of the sample. The test is terminated if any water percolates along the side wall or leaks from the pipe.

The test is not begun until the air bubbles have been sufficiently evacuated. The evacuation is conducted by allowing flow through the test channel but repeatedly agitating the water head at the outlet. To the purpose, a big rod with a diameter just less than the inner diameter of the outlet pipe is inserted into the outlet to drain the water and then is pulled out suddenly to drop the water head at the outlet. The process is repeated until no visible air bubbles evacuate from the top surface of the sample. The test then begins and the starting water head differences are set as 30,40 , and $50 \mathrm{~cm}$. Two gauge glasses are jointed to the water inlet and outlet to aid the reading of the water head difference across the sample Figure 2(b).

The water head flowing through the previous concrete sample does not occur in a quasi-steady state. Recording the water head and its associated time by manual labor may introduce considerable experimental errors. In this study, the falling head process is recorded as a video, which is then converted to JPG images in frames. For each specimen, videos recording the water head-falling processes are recorded three times. Any two desired heads, for example, $20 \mathrm{~cm}$ and $2 \mathrm{~cm}$, 


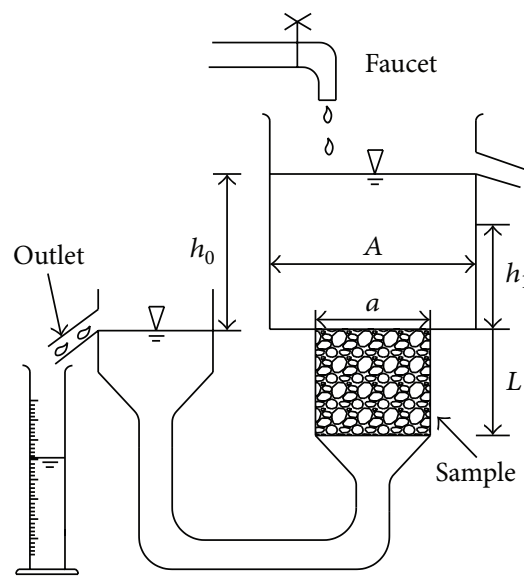

(a)

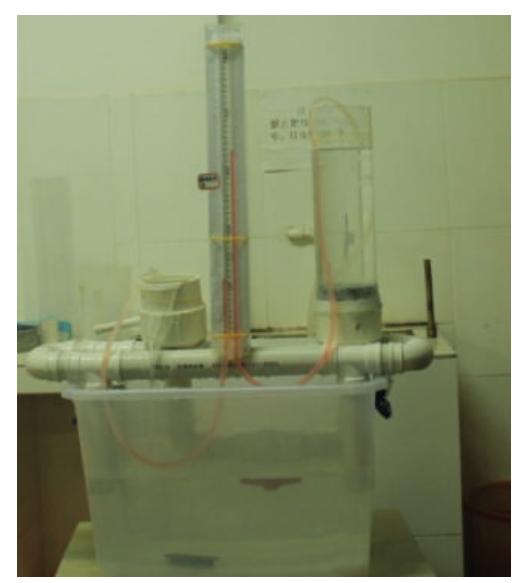

(b)

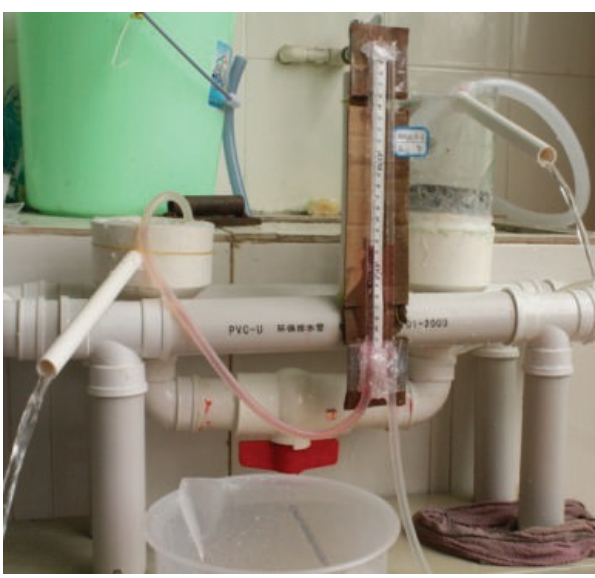

(c)

Figure 2: Apparatuses used to measure the permeability of pervious concrete: (a) schematic outline, (b) falling water head setup, and (c) constant water head setup.

can be picked up from the frames; the time spacing between two frames is the associated time interval.

2.2.2. Constant Head Method. The CHM adopts the same sample-preparing protocols as the FHM does. The CHM to determine the permeability of pervious concrete is assembled as in Figure 2(c). A pipe is installed in the inlet to drain the additional water supplies from the faucet and to support a steady water head above the sample. The water head difference can be adapted as any desired level by adjusting the pipe to the designed elevation. The water drained from the outlet is collected by a container. Supposing that a volume of drained water $Q\left(\mathrm{~m}^{3}\right)$ is collected during the time interval $\Delta t$, the permeability of the sample can be computed by

$$
K_{\text {con }}=\frac{\mu Q L}{\rho g h A \Delta t} .
$$

Equations (2) and (3) compute the apparent permeability in a unit of $\mathrm{m}^{2}$, while road engineers may be more interested in the water-flowing rate through the pervious concrete, $k(\mathrm{~m} / \mathrm{s})$. The correlation of these two parameters is

$$
k=\frac{\rho g}{u} K
$$

where $K$ can be either $K_{\text {con }}$ or $K_{\text {fal }}$.

\section{Experimental Results and Discussion}

3.1. Measured Permeability. Figure 3 plots the permeability versus the applied water pressure on the sample. The water permeability of the pervious concrete cores ranges from 0.05 to $4 \mathrm{~cm} / \mathrm{s}$, representing the expected water permeability of permeable concrete. For the CHM, the permeability is measured under different water heads on the sample. For each recording video taken during the FHM, the permeability is computed by estimating the water heads dropping from $20 \mathrm{~cm}, 16 \mathrm{~cm}, 12 \mathrm{~cm}, 8 \mathrm{~cm}$, and $5 \mathrm{~cm}$ to $2 \mathrm{~cm}$ heads, respectively. For an individual water falling process (e.g., 20 to $2 \mathrm{~cm}$ ), the water permeability estimated from different recording videos is well agreed upon, validating the consistency during the measurement.

The permeability measured from the FHM is somewhat greater than that from the CHM (Figure 3). The difference ranges from 0 to $0.005 \mathrm{~cm} / \mathrm{s}$, depending on the sample and on the selection of the starting head and finishing head. The difference increases as the pressure increases. If the permeability measured from the FHM is estimated at a lower pressure (e.g., from 5 to $2 \mathrm{~cm}$ ), it becomes better close to the permeability measured from the CHM (Figure 3).

3.2. Measured Permeability and Applied Pressure. The nonlinear correlation between the applied pressure and apparent permeability infers that the water permeability of pervious concrete does not obey the Klinkenberg effect. To verify this inference, we examine if the experimental data set follows the Klinkenberg effect. According to the Klinkenberg effect, the permeability of porous media increases linearly with the reciprocal mean pressure but is independent of pressure difference [12]:

$$
k=k_{v}\left(1+\frac{b}{p_{m}}\right)
$$

where $b(\mathrm{~Pa})$ is the Klinkenberg coefficient; $k_{v}(\mathrm{~m} / \mathrm{s})$ is the material's intrinsic permeability that can be measured when the applied pressure approaches infinity; $p_{m}$ is the mean pressure. In the water permeability test, it is

$$
p_{m}=\frac{\left(p_{0}+p_{0}-\rho g h\right)}{2},
$$

where $p_{0}$ is the atmospheric pressure, $1.03 \times 10^{5} \mathrm{~Pa}$. Substituting (6) into (5) and noting that $\rho g h / 2 \ll p_{0}$, one can get

$$
k=k_{v}\left(1+b p_{0}\right)\left(1+\frac{\rho g h}{2\left(1+b p_{0}\right)}\right) \text {. }
$$






$\square$ Constant head

$\diamond$ Falling head

(a) $k$ and $P(\operatorname{mix} 1$, number 1$)$

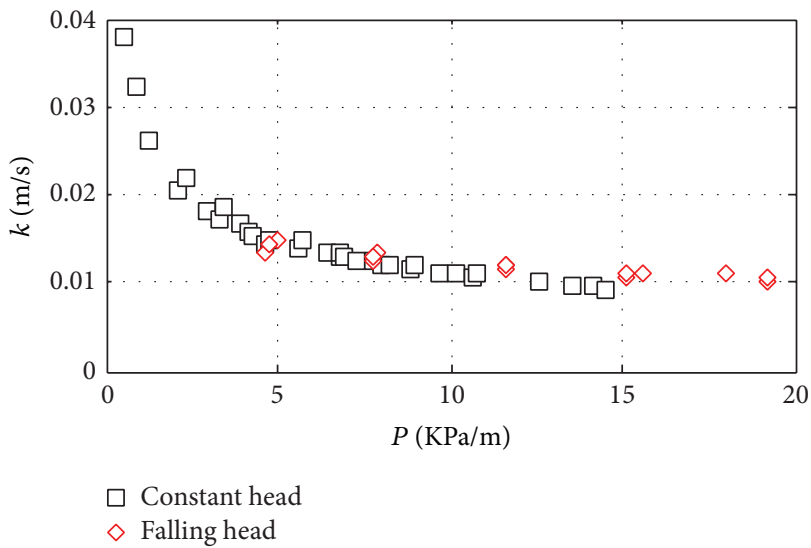

(c) $k$ and $P$ (mix 2, number 1$)$



(b) $k$ and $P$ (mix 1 , number 2$)$

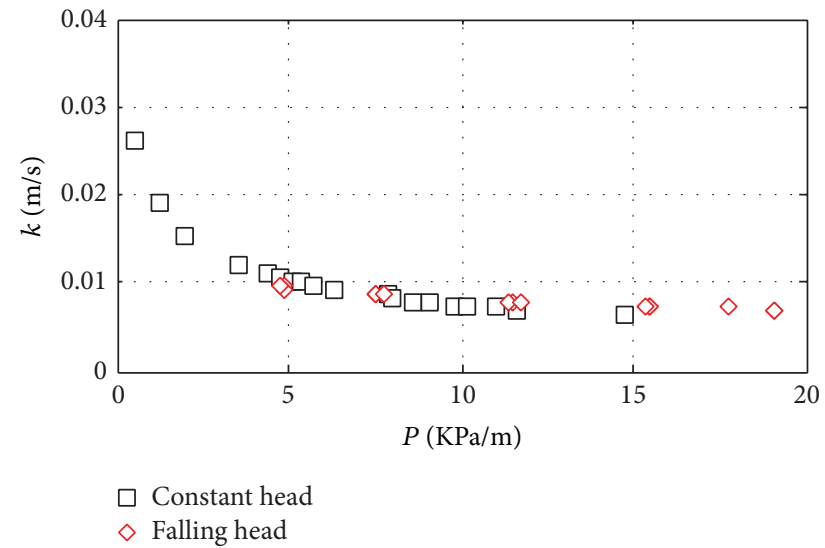

(d) $k$ and $P$ (mix 2, number 2)

FIGURE 3: Measured permeability under different pressure.

TABLE 1: The Klinkenberg coefficient and basic properties of the pervious concrete samples.

\begin{tabular}{lcccccccc}
\hline \multirow{2}{*}{ Sample } & \multicolumn{2}{c}{$k_{v}$} & \multicolumn{2}{c}{$b(\mathrm{~Pa})$} & \multicolumn{2}{c}{$R^{2}$} & Porosity $(\%)$ & Density $\left(\mathrm{kg} / \mathrm{m}^{3}\right)$ \\
\hline Mix 1 & & CHM & FHM & CHM & FHM & CHM & FHM & \\
$\quad$ Number 1 & 0.0148 & 0.0162 & 0.2194 & 0.2616 & 0.981 & 0.960 & $31.59 \%$ & 1731 \\
$\quad$ Number 2 & 0.0096 & 0.0099 & 0.2456 & 0.3110 & 0.906 & 0.749 & $28.19 \%$ & 1767 \\
Mix 2 & & & & & & & & \\
$\quad$ Number 3 & 0.0090 & 0.0096 & 0.3137 & 0.2393 & 0.965 & 0.884 & $26.59 \%$ & 1802 \\
$\quad$ Number 4 & 0.0059 & 0.0064 & 0.3472 & 0.2462 & 0.970 & 0.969 & $25.64 \%$ & 1828 \\
\hline
\end{tabular}

According to (7), the apparent permeability should correlate linearly with the applied pressure. This linear correlation is not observed in the measured permeability (Figure 3), confirming that the permeability of pervious concrete fails to obey the Klinkenberg effect.

Here, we replace the applied mean pressure with the differential pressure in (5) as showed:

$$
k=k_{v}\left(1+\frac{b}{\rho g h}\right) .
$$

The apparent permeability will correlate linearly with the reciprocal applied pressure difference if (8) holds. Figure 4 plots the apparent permeability and the different reciprocal pressure. Table 1 tabulates the statistic coefficients $\left(k_{v}, b\right)$ and lists the porosity and density for comparison. The permeability of pervious concrete obeys (8), as vindicated in Figure 4 and $R^{2}$ in Table 1 . The intrinsic permeability $k_{v}$ is the permeability when the pressure difference approaches infinity. Considering that an infinite pressure difference is equal to an infinite mean pressure $\left(p_{1}+p_{2} \rightarrow \infty\right.$ is equal to $p_{1}-p_{2} \rightarrow \infty$ ), the permeability $k_{v}$ in (5) has the same meaning and magnitude as the permeability $k_{v}$ in (8); both are the intrinsic permeability.

The intrinsic permeability is observed increasing with the permeability; a trend has been observed in previous reports $[13,14]$. The coefficient $b$ is found to be decreasing 


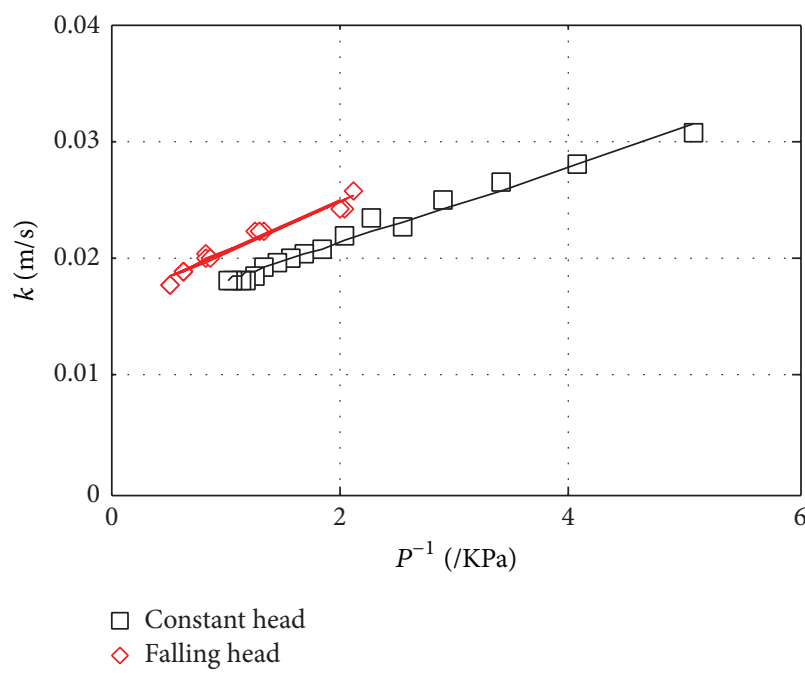

(a) $k$ and $1 / P(\operatorname{mix} 1$, number 1$)$

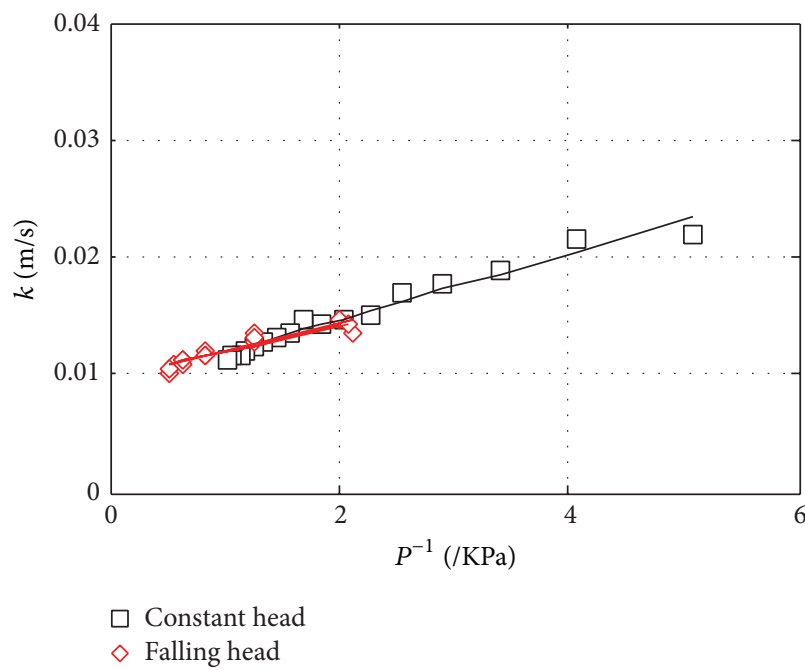

(c) $k$ and $1 / P(\operatorname{mix} 2$, number 1$)$

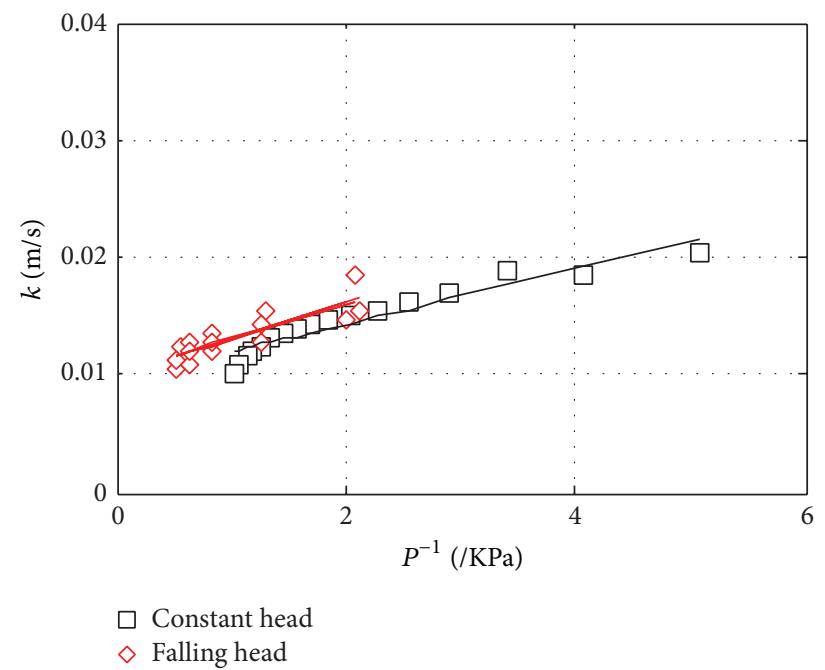

(b) $k$ and $1 / P($ mix 1 , number 2$)$

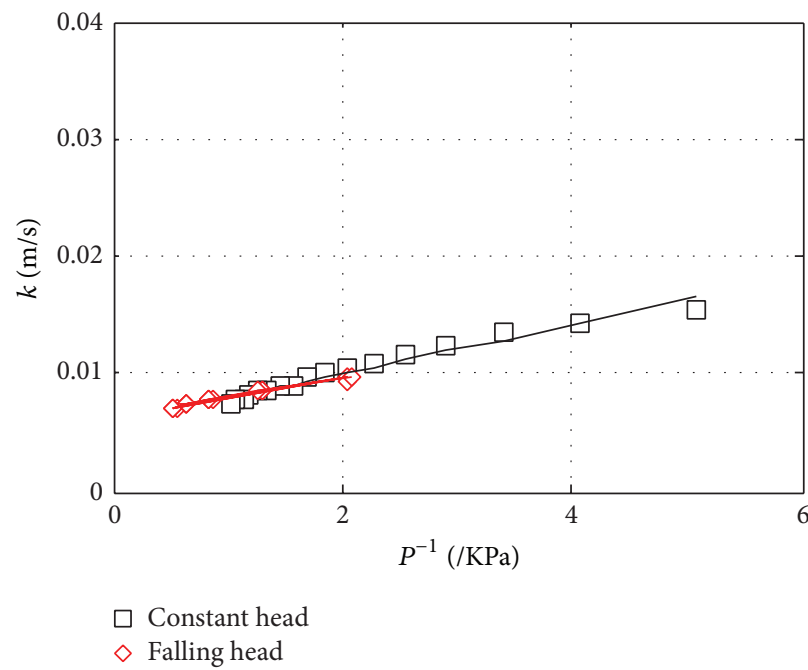

(d) $k$ and $1 / P(\operatorname{mix} 2$, number 1$)$

FIGURE 4: Klinkenberg Effect of the measured permeability of pervious concrete.

with the increase of the density and porosity, suggesting less dependence of the apparent permeability at high porous concrete.

\subsection{Difference between Constant Head and Falling Head Meth-} ods. As the apparent permeability estimated from the CHM and the FHM exhibits differences, it merits examining the mechanism behind. From both methods, the water flowing rate through the sample $v(\mathrm{~m} / \mathrm{s})$ is computed according to Darcy's law:

$$
v=-\frac{K}{\mu} \nabla P,
$$

where $\nabla P$ is the pressure gradient across the sample $(\mathrm{Pa} / \mathrm{m})$. According to the Bernoulli equation, the water gradient applied across the sample is proportional to the water head difference $h$; that is,

$$
\nabla P=\frac{\rho g h}{L} .
$$

For the CHM, the flowing rate through the sample can be estimated from the water collected by the container and is computed by

$$
v=\frac{Q}{A \Delta t} .
$$

Substituting (10)-(11) into (9), one has (3). It means the CHM estimates the permeability of pervious concrete by assuming a laminar flow.

For the FHM, the water flow through the sample obeys the mass conservation law and follows

$$
v \times A=\frac{d h}{d t} \times a .
$$

Substituting (10) and (12) into (9), one has

$$
\frac{d h}{h}=-\frac{\rho g K A}{\mu L a} d t .
$$


If the permeability $K$ is independent of the applied pressure (h), (13) can be integrated directly and the integral is the same as (2). This means that, in addition to the assumption of a laminar flow, the FHM also assumes that the apparent permeability is independent of the applied pressure.

In (2), any two water heads at any associated interval can be selected to estimate the permeability of previous concrete under the FHM test. This selection would not influence the measured permeability if the permeability $K$ is constant.

However, the selection does influence the estimated water permeability, as substantiated in Figures 3 and 4. The water permeability of permeable concrete tends to decrease with the increase of the pressure. The correlation obeys (8). Substituting (8) into (13), one can integrate (13) and solve for the permeability, as expressed in

$$
K_{\mathrm{fal}}=\frac{\mu L a}{\rho g A \Delta t} \ln \left(\frac{h_{0}+b / \rho g}{h+b / \rho g}\right) .
$$

Equation (14) is equal to (1) only if $b$ vanishes. In the case of $b=0$, the slope of the linear line in Figure 4 is zero or the apparent permeability is independent of the imposed pressure difference. As natural logarithm is a monotonically increasing function, neglecting the term of $b / \rho g$ overestimates the right-hand side of (14) and thus overestimates the water permeability. That is why the estimated permeability from the FHM tends to be higher than that from the CHM (Figure 3).

Therefore, the FHM presumes the apparent permeability is independent of the applied pressure difference but the CHM does not. This assumption introduces difference to the permeability measured from the two methods.

\section{Conclusions}

Falling head and constant head methods are, respectively, used to measure the permeability of pervious concrete samples. The measured permeability is found to be decreasing with the applied pressure difference on the sample but does not obey the Klinkenberg effect. The permeability is found to be increasing linearly with the reciprocal water pressure, similar to the Klinkenberg effect.

The permeability measured by the CHM is lower than that by the FHM. The selection of the starting and finishing water heads from the FHM strongly affects the calculated permeability. The difference decreases as the water head approaches zero. Due to this correlation, the water permeability of permeable concrete should be reported with the applied pressure and the associated testing method.

\section{Conflict of Interests}

The authors declare that there is no conflict of interests regarding the publication of this paper.

\section{Acknowledgments}

This work is supported by National Natural Science Foundation of China (Grant nos. 51268005 and 51308135) and by the
Scientific Research Foundation of Guangxi University (Grant no. XGZ130237). The authors are indebted to their graduate students Yingpu Xie, Ting Bao, and Yumei Wang for the lab works.

\section{References}

[1] ASTM International, "Standard test method for permeability of Granular soils (constant head)," ASTM D2434-68, ASTM International, West Conshohocken, Pa, USA, 2000.

[2] ASTM International, ASTM-D5048-03, Standard Test Method for Measurement of Hydraulic Conductivity of Saturated Porous Materials Using a Flexible Wall Permeameter D4084-03, ASTM International, West Conshohocken, Pa, USA, 2003.

[3] S. B. Park, B. Lee, J. Lee, and Y. I. Jang, "A study on the seawater purification characteristics of water-permeable concrete using recycled aggregate," Resources, Conservation and Recycling, vol. 54, no. 10, pp. 658-665, 2010.

[4] T. Tho-In, V. Sata, P. Chindaprasirt, and C. Jaturapitakkul, "Pervious high-calcium fly ash geopolymer concrete," Construction and Building Materials, vol. 30, pp. 366-371, 2012.

[5] Y. Qin, H. Yang, Z. Deng, and J. Zhang, "A simplified model for computing pollutants release from granular pavement base to local aquifer," Environmental Earth Sciences, vol. 75, no. 5, pp. 1533-1540, 2014.

[6] R. Zaharieva, F. Buyle-Bodin, F. Skoczylas, and E. Wirquin, "Assessment of the surface permeation properties of recycled aggregate concrete," Cement and Concrete Composites, vol. 25, no. 2, pp. 223-232, 2003.

[7] J. P. Coughlin, C. D. Campbell, and D. C. Mays, "Infiltration and clogging by sand and clay in a pervious concrete pavement system," Journal of Hydrologic Engineering, vol. 17, no. 1, pp. 6873, 2011.

[8] M. Sonebi and M. T. Bassuoni, "Investigating the effect of mixture design parameters on pervious concrete by statistical modelling," Construction and Building Materials, vol. 38, pp. 147-154, 2013.

[9] O. Deo, M. Sumanasooriya, and N. Neithalath, "Permeability reduction in pervious concretes due to clogging: experiments and modeling," Journal of Materials in Civil Engineering, vol. 22, no. 7, pp. 741-751, 2010.

[10] C. Lian and Y. Zhuge, "Optimum mix design of enhanced permeable concrete-an experimental investigation," Construction and Building Materials, vol. 24, no. 12, pp. 2664-2671, 2010.

[11] F. Montes, S. Vlavala, and L. M. Haselbach, "A new test method for porosity measurements of portland cement pervious concrete," Journal of ASTM International, vol. 2, no. 1, pp. 1-13, 2005.

[12] L. J. Klinkenberg, "The permeability of porous media to liquid and gases," in Drilling and Production Practice, American Petroleum Institute, New York, NY, USA, 1941.

[13] J. Kevern, K. Wang, M. T. Suleiman, and V. R. Schaefer, Mix Design Development for Pervious Concrete in Cold Weather Climates, Iowa State University, Ames, Iowa, USA, 2005.

[14] F. Montes and L. Haselbach, "Measuring hydraulic conductivity in pervious concrete," Environmental Engineering Science, vol. 23, no. 6, pp. 960-969, 2006. 

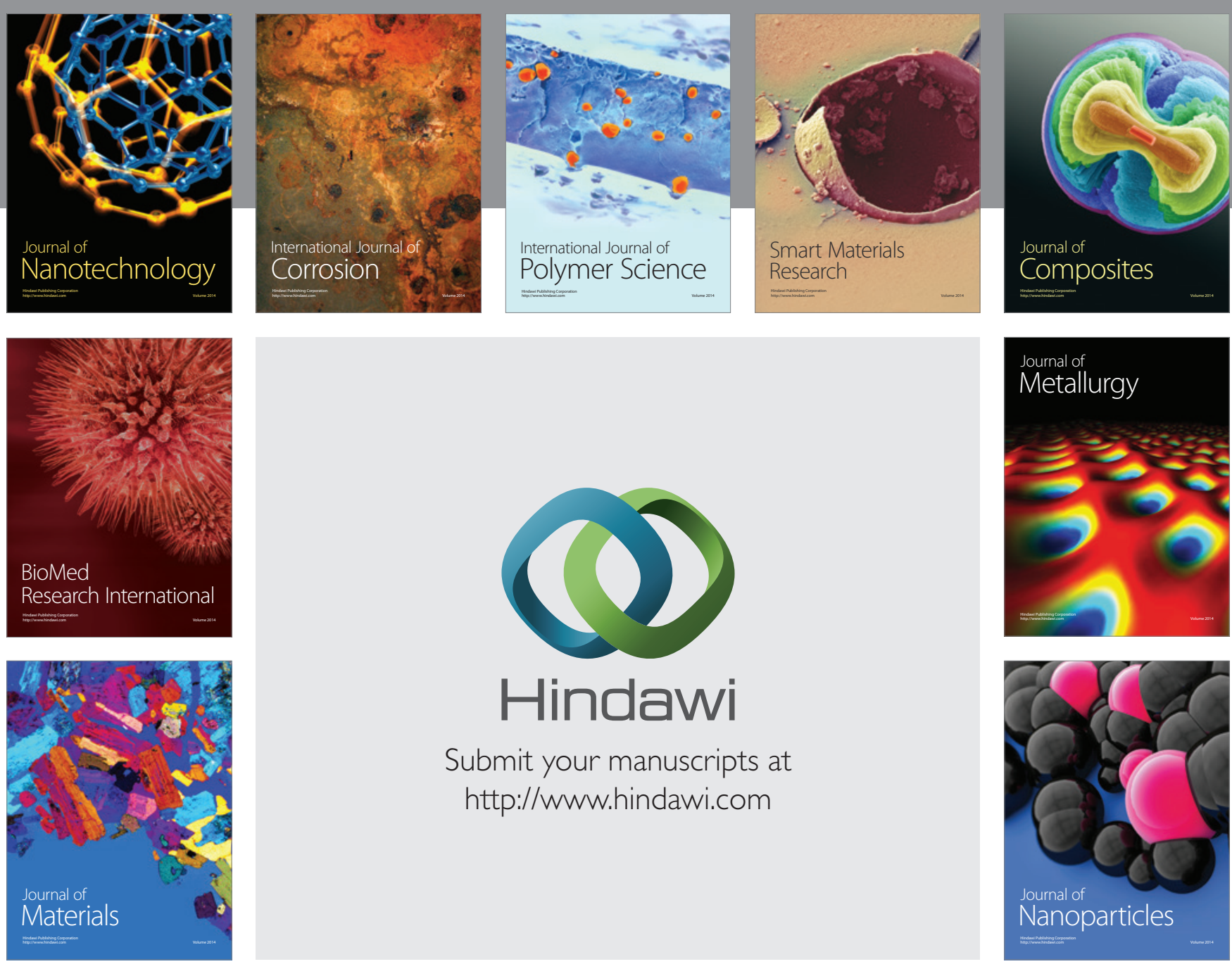

Submit your manuscripts at http://www.hindawi.com
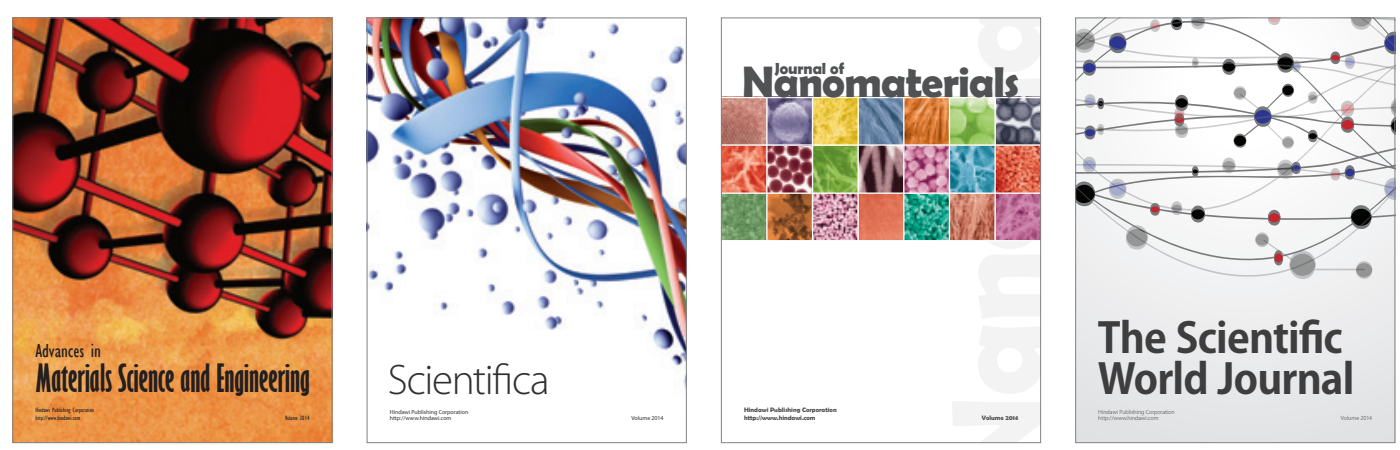

\section{The Scientific World Journal}
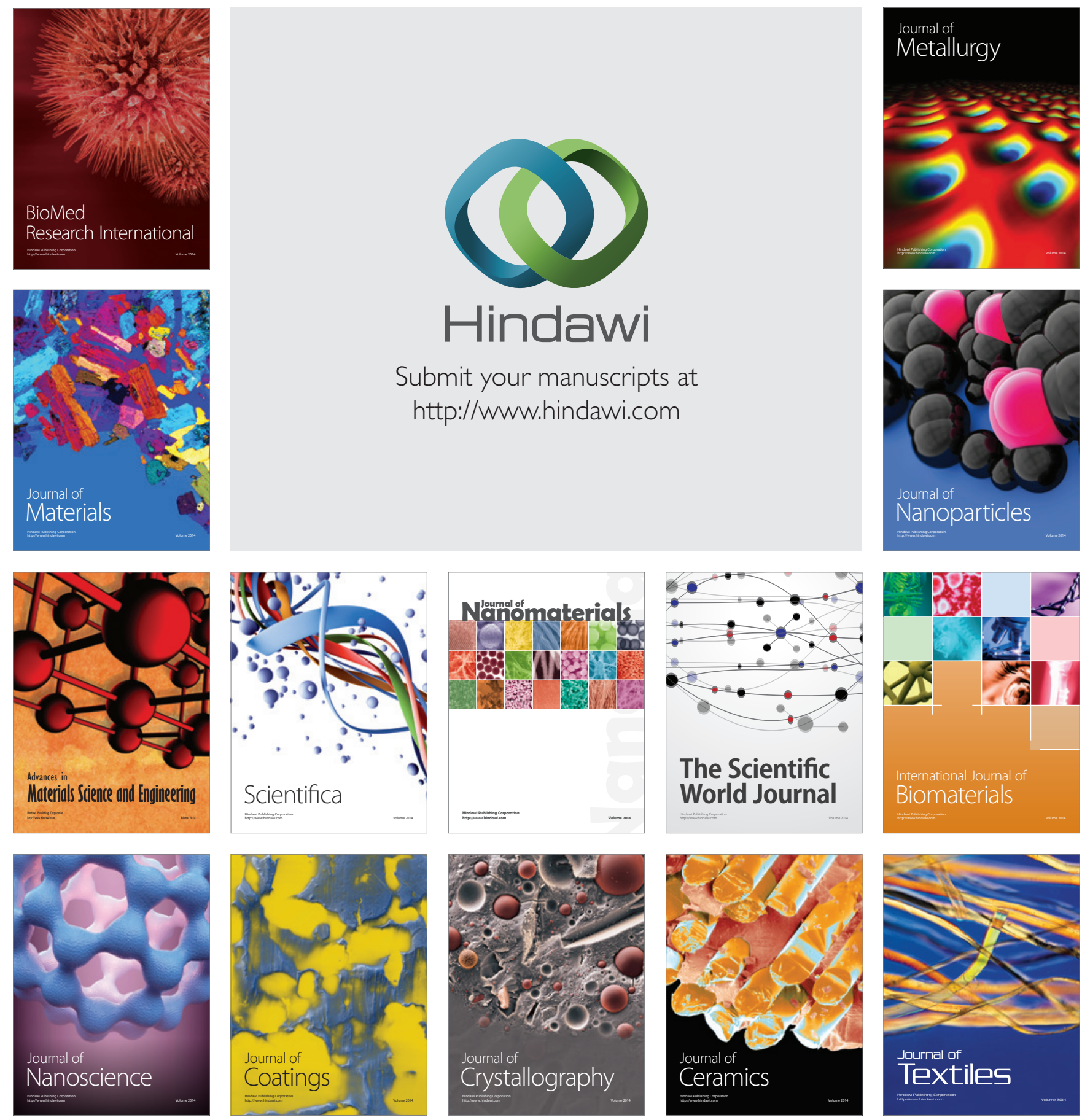